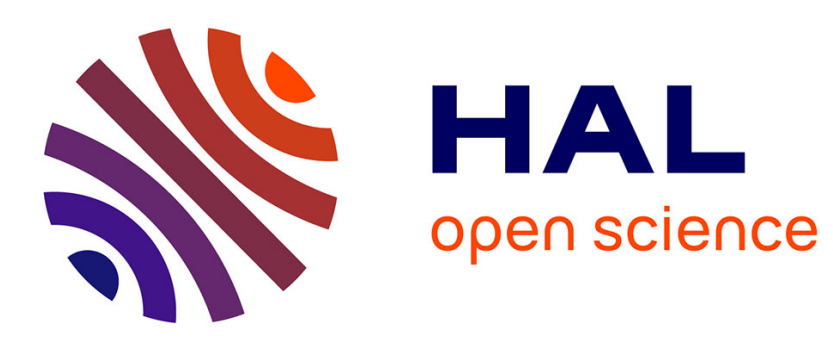

\title{
Prémices d'une éducation sentimentale : l'intimité masculine dans les collèges (1815-1848)
}

\author{
Gabrielle Houbre
}

\section{To cite this version:}

Gabrielle Houbre. Prémices d'une éducation sentimentale : l'intimité masculine dans les collèges (1815-1848). Romantisme: la revue du dix-neuvième siècle, 1990, 68, pp.9-22. 10.3406/roman.1990.6122 . hal-01353784

\section{HAL Id: hal-01353784 \\ https://hal.science/hal-01353784}

Submitted on 16 Aug 2016

HAL is a multi-disciplinary open access archive for the deposit and dissemination of scientific research documents, whether they are published or not. The documents may come from teaching and research institutions in France or abroad, or from public or private research centers.
L'archive ouverte pluridisciplinaire HAL, est destinée au dépôt et à la diffusion de documents scientifiques de niveau recherche, publiés ou non, émanant des établissements d'enseignement et de recherche français ou étrangers, des laboratoires publics ou privés. 


\section{Prémices d'une éducation sentimentale : l'intimité masculine dans les collèges (1815-1848)}

In: Romantisme, 1990, n68. Amours et société. pp. 9-22.

Citer ce document / Cite this document :

Houbre Gabrielle. Prémices d'une éducation sentimentale : l'intimité masculine dans les collèges (1815-1848). In: Romantisme, 1990, n68. Amours et société. pp. 9-22.

doi : 10.3406/roman.1990.6122

http://www.persee.fr/web/revues/home/prescript/article/roman_0048-8593_1990_num_20_68_6122 
Gabrielle HOUBRE

Prémices d'une éducation sentimentale :

l'intimité masculine dans les collèges (1815-1848)

* Du collège chacun déteste

La discipline et les rigueurs ;

Et plus tard, pourtant, il en reste

Un doux souvenir dans les cours *1.

Ces couplets, chantés lors du banquet annuel des anciens élèves du collège Louis-Le-Grand, en 1850, mettent en évidence un paradoxe caractéristique des témoignages sur les établissements d'enseignement public ou privé : tous, ou presque tous, s'accordent à dénoncer la claustration, le délabrement, l'insalubrité, l'extrême inconfort des lieux, l'hygiène exécrable, l'alimentation pitoyable, la sévérité de la discipline et celle, plus grande encore, des punitions qui sanctionnent la moindre entorse à la règle ${ }^{2}$. Et cependant tous, ou presque tous, éclaircissent la tonalité sombre des années passées au collège par l'évocation lumineuse des amitiés nouées sur ses bancs ${ }^{3}$.

Désiré Nisard, dans un chapitre de ses Souvenirs et notes biographiques consacré à ses amitiés de collège, aborda le sujet devenu argument d'une discussion polémique sur le bien-fondé de l'internat de collège :

Les novateurs qui s'attaquent à notre vieux système de collèges à internat ne laissent pas d'avouer que parmi des inconvénients, selon eux très graves, ils offrent quelques avantages [...]. Parlant des inconvénients, et de ce qu'ils appellent * les horreurs de l'internat $*$, ils y épuisent tout le vocabulaire de la langue pénitentiaire [...]. S'agit-il des avantages ? Ils sont ou insaisissables ou contestables [...]. Il en est un pourtant dont ils conviennent honnêtement, et qu'ils reconnaissent en termes explicites : c'est qu'il se forme dans les collèges à internat « des amitiés plus intimes et plus durables que celles des jeunes gens du même âge restés libres ${ }^{4}$.

Et Nisard de s'en prendre à l'explication avancée par ces « novateurs », pour qui ces amitiés doivent leur intensité et leur force « à ce qu'on a fait campagne, et souffert ensemble " ${ }^{4}$, dénonciation directe de l'administration de l'enseignement public que le respectueux universitaire et homme politique qu'il est, ne peut accepter. Nisard se refuse à réduire les élans affectifs de deux condisciples à une solidarité dictée par les vicissitudes quotidiennes inhérentes à la vie de collégien. Considérant sa propre expérience, sa relation privilégiée avec son ami Trancart au collège de Sainte-Barbe, il invoque l'exemple de Montaigne et La Boétie et s'en tient au fameux commentaire : « je l'aymois, parce que c'estait luy, parce que c'estait moy ${ }^{5}$. 
Alain Corbin, s'intéressant à l'intimité de l'individu dans Histoire de la vie privée, a attiré l'attention des historiens sur ces liaisons passionnées d'adolescents : « Les historiens du XIXe siècle ont par trop négligé le poids de l'amicalité et les plaisirs de la confidence. C'est risquer de mal comprendre l'histoire de ce temps sensible, à tous les niveaux, au déploiement des affinités électives " ${ }^{6}$. A regarder de près, on ne saurait trop dire de ces relations exacerbées, qui nous sont révélées par les correspondances, journaux intimes, mémoires, souvenirs et autres écrits à caractère autobiographique, si elles sont plus amicales qu'amoureuses. Au-delà des simples sentiments de sympathie réciproque, ces liaisons trouvent leur spécificité dans une relation conflictuelle. Elle met en jeu, pour l'adolescent, des éléments de nature différente, tels l'influence de son éducation familiale, le poids des exigences sociales, la période physiologique et psychologique caractéristique de la puberté, ainsi que le lieu, non moins caractéristique, où se déroule, pour l'essentiel, ce conflit : le collège.

L'enfant qui franchit pour la première fois la porte du collège, généralement entre huit et douze ans, porte l'empreinte d'une première éducation familiale. Celle-ci, surtout maternelle, est régie par une volonté de moralisation, obsessionnelle au XIXe siècle, qui, perceptible sous la Restauration, devient tapageuse sous la Monarchie de Juillet. Les pédagogues ecclésiastiques eurent soin d'annexer ce concept d'éducation morale pour le seul profit de l'éducation religieuse ${ }^{7}$, tandis que les pédagogues laïques le prônèrent au nom de l'éducation sociale $^{8}$. Dans les deux cas il restait sous-tendu par une même crainte du sexe de l'enfant, crainte que Michel Foucault a mise en avant :

[une] double affirmation [est faite] que presque tous les enfants se livrent ou sont susceptibles de se livrer à une activité sexuelle; et que cette activité sexuelle étant indue, à la fois « naturelle * et « contre nature *, elle porte en elle des dangers physiques et moraux, collectifs et individuels ; les enfants sont définis comme des êtres sexuels « liminaires $»$, en deçà du sexe et déjà en lui, sur une dangereuse ligne de partage ${ }^{9}$.

Dès lors il devient vital, pour une société traumatisée par le tableau morbide des ravages de l'onanisme dépeint par le Dr Tissot (L'Onanisme, 1760 édition française), de gérer « ce germe sexuel, précieux et périlleux, dangereux et en danger » ${ }^{9}$. C'est la mission occulte conférée à l'éducation morale que d'agir en contre-éducation sexuelle :

Les influences morales, c'est-à-dire celles qui par l'esprit arrivent aux sens, prédisposent souvent les jeunes sujets aux abus dont nous traitons [masturbation] [...] aussi l'éducation morale, cette éducation qui consiste à écarter de la jeunesse certaines impressions, à faire que celles qui doivent nécessairement arriver, n'agissent que lorsque leur temps est venu doit-elle être considérée comme un des moyens les plus efficaces pour prévenir l’abus prématuré des plaisirs vénériens ${ }^{10}$.

Médecins et pédagogues s'entendent à prêcher des dispositions précises permettant de « préserver » l'enfant. Sa sexualité, mais aussi son affectivité sont l'objet de la plus extrême attention : il s'agit d'abord de garantir les conventions et la bienséance socio-familiales de tout écart ressenti comme une agression. L'enfant devient l'objet d'un véritable complexe sécuritaire. Le thème des « mauvaises influences » à traquer sans répit s'enracine durablement dans le 
discours : l'environnement familier de l'enfant est soumis à une surveillance minutieuse. La domesticité - * corruptrice de l'enfance »-, à laquelle s'attache une suspicion tenace depuis Rousseau (qui déjà voulait « élever Emile à la campagne, loin de la canaille des valets " ${ }^{11}$ ), est la première mise en cause. Il est formellement recommandé de ne pas laisser « jouer ensemble des enfants des deux sexes dans le plus grand pêle-mêle $»$, tant on redoute des conséquences funestes :

Certaines émotions se glissent entre eux ; vagues, obscures dans l'origine, elles prennent ensuite plus de consistance ; le foyer d'où elles partent s'échauffe, la curiosité s'y attache, et bientôt le secret des plaisirs solitaires est trouvé ${ }^{12}$.

La curiosité enfantine, qui plus que jamais est un bien vilain défaut, est vivement appréhendée : " les jeunes sujets peuvent encore saisir, sous le toit paternel, des notions dangereuses sur les différences matérielles qui séparent les sexes, et sur d'autres qui en sont la conséquence » ${ }^{12}$. Pour échapper à ce péril, on se préoccupe de censurer autant que possible la connaissance sensorielle de l'enfant. Lui sont formellement interdits livres, images, tableaux, sculptures pouvant susciter vagabondages imaginaires et dérèglements physiques. A cet égard, l'exemple de Jules Barbey d'Aurevilly est typique de la situation que l'on cherche à éviter : petit garçon, échappant aisément à la surveillance négligente de sa mère, il aimait à se planter devant un buste féminin, relégué dans un coin du salon familial, en proie à de troublantes songeries. Dans un poème glorificateur composé alors qu'il était sexagénaire, Barbey d'Aurevilly désignera ce buste, tôt métamorphosé en objet érotique, comme le prélude pédagogique à son éducation sentimentale et sexuelle ${ }^{13}$.

Pour annihiler toute propension à la curiosité de l'enfant, le couple parental est instamment prié d'adopter la plus stricte réserve dans son comportement en sa présence. La sexualité perd droit de cité hors de la chambre conjugale ${ }^{14}$. L'affectivité entre époux ne peut s'exprimer à travers des gestes ou des mots trop évocateurs, celle existant entre parents et enfants ne doit pas prêter à des sensations et des pulsions physiques trop ardentes, de même celle qui attache les enfants aux autres figures familiales. Jules Michelet, dans ses Ecrits de jeunesse, se souvient parfaitement de ses visites de petit garçon à sa grand-mere : " $j$ 'y jouais avec ma tante et le jeu, toujours innocent dans ses commencements, ne laissait pas de m'animer beaucoup ${ }^{15}$.

Ce processus de protection contre les mauvaises influences qui, agencé dès les premières années de l'enfance, ne prête guère à l'épanouissement individuel, va se renforcer considérablement au moment de la période pubertaire, période redoutée entre toutes. « La puberté est considérée comme une crise d'identité potentiellement dangereuse pour lui (l'adolescent) comme pour la société » remarque Michelle Perrot ${ }^{16}$. L'effarement face à la masturbation, dont Alain Corbin a rappelé qu'il reposait avant tout « sur le fantasme de la perte, sur la nécessité de gérer toute dépense et donc d'échafauder une saine économie spermatique ${ }^{17}$, atteint ici son paroxysme. Les médecins et le clergé, dans une exceptionnelle union sacrée, dénoncent vigoureusement le fléau, et diffusent à profusion l'image morbide de « l'onaniaque » :

Voyez-vous ces lèvres pâles, ces pommettes saillantes, ces joues creuses et comme sillonnées par le feu des passions qui le dévoraient? Un teint jaune et 
livide a remplacé les fraîches couleurs de la vertu. Plus de regards vers le ciel ; son œil terne, environné d'un cercle noir, regarde stupidement la terre. Une voix grêle et rauque sort avec peine de ce corps décharné, comme d'entre les ossements d'un squelette. Sa respiration est courte, sèche, pénible ; sa démarche est incertaine, il chancelle et va tomber pour jamais : il ne lui manque qu'une fosse ouverte ${ }^{18}$.

Ces mises en scène ne sont pas sans effet sur l'imaginaire des jeunes garçons, l'administration des collèges n'hésitant pas, le cas échéant, à propager des rumeurs alarmistes. Ainsi, au collège de Sorèze en 1820, le décès d'un élève excite les esprits :

C'est ses polissonneries qui l'ont tué. Il y a deux jours qu'épuisé par la fatigue, il retomba dans ses habitudes ; le lendemain il ne put se lever ; on le porta à l'infirmerie ; toutes les fois qu'il voulait se relever, il retombait, les reins pliaient; enfin après avoir passé une fort mauvaise nuit, il est mort ce soir victime de son libertinage. Voilà un exemple terrible,

raconte, impressionné, un élève à son ancien condisciple ${ }^{19}$.

Volontiers intimidé par ces procédés, l'adolescent - en pleine « révolution » physiologique $^{20}$ - qui tenterait de se mettre au fait des questions sexuelles, ne trouverait guère de recours au sein de sa famille, tant le tabou y est vivace.

La pression qui s'exerce sur l'adulte pour qu'il « privatise * toutes les manifestations pulsionnelles et plus spécialement sexuelles, le « voile du silence $\nsim$, les restrictions imposées par le société à sa liberté d'expression, la mise au ban de la plupart des mots ayant trait à la vie pulsionnelle, symbole des pressions psychologiques qui l'accablent, tout cela contribue à ériger autour de l'adolescent un * mur de mystère * plus ou moins infranchissable,

écrivait déjà en 1939 Norbert Elias ${ }^{21}$. Rares sont les parents, comme la mère de Jules Favre ${ }^{22}$, qui risquent l'esquisse d'une éducation sexuelle. Le dialogue n'a pas lieu non plus entre frère et sœur, même quand ceux-ci sont particulièrement proches ; seul un frère aîné peut initier son cadet aux mystères de la sexualité, comme le fit Edmond pour Jules de Goncourt ${ }^{23}$.

Le collège, dans ses principes administratifs et pédagogiques, reprend pour l'essentiel les grandes directions de l'éducation familiale ${ }^{24}$. Pour s'assurer une clientèle, une bonne tenue morale est aussi nécessaire à un établissement que la réussite de ses élèves aux Concours. Aussi l'administration met-elle tout en œuvre pour sauvegarder cette moralité : division des collégiens en groupes autonomes - petits, moyens, grands - avec études, réfectoires, parloirs et cours de récréation séparés, repli sur le collège pour éviter de pernicieuses influences extérieures. Les visites de parents, considérées comme préjudiciables à « l'équilibre » de l'enfant, ne sont guère encouragées. Les parenthèses extrascolaires étant jugées perturbatrices, on tente de dissuader les familles de reprendre leur progéniture durant les vacances.

L'externe, qui echappe à la claustration totale du collège et pratique un va-etvient incessant avec le monde et ses tentations, est l'objet d'une forte prévention : c'est par lui que s'infiltrent les rumeurs affriolantes sur la sociabilité à venir, c'est par lui que sont introduites les lectures ou les images aguicheuses, propres à enflammer les sens des écoliers reclus. De cela, l'administration se méfie avant 
tout et cherche à se garantir par une surveillance omniprésente. Où se trouve l'adolescent, se trouve également l'œil du surveillant, et ce jusque dans les latrines où l'on prétend maintenir « les mœurs [...] à l'abri de toute alarme sous les yeux d'une active surveillance ${ }^{25}$... En 1857 Flaubert, dans une lettre à son ami et ancien condisciple Bouilhet, évoque non sans nostalgie « ces braves latrines où l'urine par flaques énormes aurait pu noyer le cheval de Préault [...] ces pauvres kiques où l'on fumait des cigarettes de maryland, et où l'on se branlait si poétiquement avec des doigts abîmés d'engelures ${ }^{26}$.

Par toutes ces mesures, et quelques autres, l'administration espérait sauvegarder la candeur de ses élèves, vou pieux entre tous. De sa classe de Rhétorique au Lycée Charlemagne (1829-1830), l'universitaire François Chon garde le souvenir de la résistance insidieuse et permanente de ses camarades aux contraintes édictées par le règlement :

Je passe donc sur les conversations habituelles dans les études et les récréations, sur les images et les livres circulant à la dérobée, sur les dessins crayonnés ou sculptés dans les endroits d'une fréquentation inévitable, effacés quelquefois par une autorité pudibonde et toujours rétablis. C'était réellement de l'instruction gratuite, obligatoire et laïque, et quelle instruction ! ${ }^{27}$.

Pour prévenir la propagation du mal, l'administration avait soin d'isoler chaque élève, de lui ôter toute occasion d'aparté prolongé avec l'un de ses pairs. Les maîtres d'étude étaient, pour ce faire, dûment chapitrés : « Ils font une grande attention aux élèves qui se recherchent pour s'isoler, pour se mettre à l'eccart, et avoir des entretiens particuliers ; ils ont soin de les ramener auprès des autres, et en vue de tout le monde ${ }^{28}$. Tout était fait pour contrarier et décourager les amitiés intimes. Avivées par les obstacles dressés et les interdits prononcés, elles ne se développaient que davantage.

Placé dans un environnement qu'il ressent ordinairement comme hostile, intégré plus ou moins bien dans une disposition nouvelle de sociabilité - qui entremêle parfois difficilement et même douloureusement les rapports avec les maîtres d'étude, les professeurs, et les autres élèves -, le jeune garçon, sevré au quotidien des affections familiales, quand elles existent, aspire à la sympathie et à la compréhension d'autrui. Désabusé, le jeune Fernand de Sassenay explique ce sentiment : « parfois cependant ma bonne mère je suis triste de ne pas trouver un cœur dans lequel je puisse répandre une foule de petites afflictions dont la vie d'un écolier est parsemée " ${ }^{20}$.

C'est le désir profond du jeune garçon que de trouver un confident, véritable compagnon d'élection, qui autorise les épanchements du cour les plus spontanés, qui rende pleinement légitime le discours du « moi ». " En quête de lui-même, ladolescent est narcissique ; il cherche son image morale et physique. Il est fasciné par le miroir », note Michelle Perrot dans Histoire de la vie privée ${ }^{30}$.

Ce miroir peut être l'autre, - l'alter ego -, capable de le connaître et de le comprendre ; les premières protestations d'amitié vont en ce sens : « J'ai besoin d'ouvrir mon cour, de m'associer à une âme qui me comprenne, et je t'ai choisi pour me consoler et pour t'aimer », dévoile Charles de Montalembert à Léon Cornudet ${ }^{31}$; « je t'ai tout dit, je t'ai ouvert mon cœur, tu me connais tout entier. Tu sais maintenant si tu veux me continuer ton amitié, la rompre ou la resserrer davantage. Quoi qu'il en soit, je t'aimerai toujours, je désirerai toujours demeurer 
et devenir de plus en plus ton ami ", énonce pour son compte Frédéric Ozanam à Auguste Materne ${ }^{32}$. Un alter ego capable aussi de lui réfléchir son image : le jeune garçon, comme s'il lui était malaisé de se livrer seul et directement à son introspection, sollicite, en le présentant essentiel à la relation, le regard critique de l'autre : « Si tu veux me continuer ton amitié, je te prie de ne point me ménager, de me donner de bons conseils et de n'être jamais flatteur » (Ozanam à Materne, 5 juin 1830). " Si tu veux me convaincre que tu accueilles favorablement mon amitié et ma confiance, j'exige de toi que tu sois inexorable sur tout ce que tu verras de répréhensible en moi et que tu m'en avertisses sans ménagement sur-lechamp » (Montalembert à Cornudet, 5 juin 1827).

Pour que l'amitié intime puisse s'épanouir pleinement avec quelque gage de solidité, il convient que chacun ait reconnu dans l'autre des sentiments et des convictions suffisamment proches pour permettre une union étroite. Balzac a évoqué, dans Louis Lambert, pareil mariage de convenance entre collegiens :

La conformité de nos goûts et de nos pensées nous rendit amis et faisants. Notre fraternité devint si grande que nos camarades accolèrent nos deux noms; l'un ne se prononçait pas sans l'autre [...]. D'autres noms offraient l'exemple d'un semblable mariage. Ainsi je demeurai pendant deux années l'ami de collège de Louis Lambert $^{33}$.

Michelet, qui pourtant se rappelle un temps probatoire aux véritables débuts de sa liaison passionnée avec Poinsot, n'exprime pas autre chose : "Ce qui peutêtre nous attira l'un vers l'autre avant que nous connussions combien nous nous ressemblions l'un à l'autre, c'était de son côté le plaisir d'entendre des histoires, du mien celui d'en conter », avant de préciser que la " conformité de pensées, de sentiments » les avait rendus inséparables ${ }^{34}$. Nisard se contente de révéler, sobrement mais explicitement, le même phénomène en avouant avoir aimé tout ce que son ami aimait ${ }^{35}$. Cornudet, en revanche, exulte en constatant l'adéquation parfaite de sa personnalité à celle de Montalembert : « je voyais, non sans un sentiment d'amour-propre et de plaisir, que, sans nous être jamais connus, nos idées religieuses, politiques, morales, etc., cadraient merveilleusement " ${ }^{36}$. Ozanam, après s'être persuadé, dans l'allégresse, que Materne et lui étaient décidément faits l'un pour l'autre, détaille leurs attitudes communes avec soulagement : « Tout ce que tu m'as dit dans ta dernière lettre sur ta bizarrerie, sur tes accès de piété, sur tes attaques ou tes éloges en fait de religion, sur tes sécheresses à la confession et à la Saint Table, sur tes pensées voluptueuses, tout cela est mon histoire, ma fidèle histoire ${ }^{37}{ }^{37}$. Ce désir frénétique de se reconnaître dans l'autre laisse assez deviner la souffrance de ce qui est tu, ferment d'angoisses et d'un processus de culpabilisation, ainsi que le bonheur de se délivrer dans un dialogue jusque-là prohibé.

C'est dans ce besoin ardent de la confidence que transparaissent le plus clairement le poids des bienséances sociales et les séquelles de l'éducation familiale : la nature des secrets échangés accuse les interdits subis depuis l'enfance; le choix de qui les reçoit respecte le clivage sexuel. Pour ces collégiens qui sentent confusément leur sexualité s'affirmer, sans savoir, pouvoir ou oser satisfaire ses exigences, il semble alors possible de décharger leurs embarras et leurs anxiétés dans l'oreille d'un égal en butte aux mêmes turpitudes. 
Ozanam, qui vécut la période pubertaire à travers une profonde cirse spirituelle, se livre ainsi à une confession angoissé, que n'a pas sur dissiper son confesseur :

des pensées criminelles, licencieuses, m’accablaient malgré moi. Je voulais les chasser, je m'en occupais trop : mon respectable confesseur me dit de ne pas m'en inquiéter et je ne puis encore y parvenir, quoiqu'elles soient aujourd'hui plus rares [...]. Il faut que je te dise encore pour ne rien te cacher que souvent ces pensées ont glissé dans mes plaisirs quelque chose de sensuel, qu'elles égarent quelquefois mon esprit et mes yeux ${ }^{38}$.

Materne ayant répondu par un discours similaire, Ozanam, apaisé, peut déclarer : « Voilà donc notre amitié cimentée, décidée ; nos confessions mutuelles en sont le gage certain. »

La coutume, à l'intérieur des établissements d'enseignement, veut que le tabou du sexe y soit systématiquement transgressé. C'est une marotte commune à nombre de collégiens que d'afficher par des plaisanteries triviales une science encore incertaine, de rappeler par des fanfaronnades incessantes l'expérience récente de leur virilité (on songe par exemple à Flaubert et à son groupe d'amis rouennais ${ }^{39}$ ).

Tous ne se reconnaissent pas dans cette approche agressive de la sexualité, de l'amour et de la femme. A côté de la salacité rituelle des conversations entre camarades, la relation intime permet de mettre en évidence ce qui est propre à chacun, et de surmonter autrement les affres de la pudeur, sentiment mis à jour par Norbert Elias :

Le conflit qui se manifeste par cette pudeur mêlée d'angoisse n'est pas seulement un conflit entre l'individu et l'opinion sociale prédominante, mais aussi entre l'individu et cette instance de son propre moi qui représente précisément cette opinion sociale. Elle est un conflit qui met en cause sa propre économie psychique : l'individu se reconnaît lui-même comme inférieur. Il craint de perdre l'amour et l'estime de ceux à l'amour et à l'estime de qui il tient ou a tenu ${ }^{40}$.

Violentée par les usages de la camaraderie, la pudeur n'en est que plus choyée entre les intimes, tout au bonheur d'effeuiller délicatement leur âme. Au cœur de leurs confidences se dessine la silhouette de la femme, prétexte aux rêves les plus éperdus.

Dissociant volontiers le sentiment amoureux des contingences sexuelles, mais non pas toujours des désirs sensuels, les adolescents s'abandonnent aux troubles délices de l'imaginaire ${ }^{41}$. A l'instar de Chateaubriand, ils s'inventent une femme idéale, sylphide composée des grâces empruntées à des jeunes filles ou à des femmes à peine entrevues. L'amour et la femme se manifestent de façon lancinante au milieu des folles aspirations à la gloire, à l'argent et à la réussite. Une lettre de Materne à son ancien condisciple Fortoul témoigne de cet état d'esprit :

priez pour moi, car je suis bien malade, et depuis longtemps, sans que mon père s'en doute, sans qu'aucun de mes amis l'ait jamais sue [sic]. Mais je n'y peux plus tenir, il faut que j'achève une confession si avancée : écoute et aie pitié. Depuis ma plus tendre enfance, les femmes ont exercé sur mon être une singulière influence ; c'est au point que je n'avais pas de plus grand bonheur, de plus douce volupté que 
d'en voir une, de mettre ma main dans les siennes, de m'asseoir sur ses genoux ; alors j'étais fasciné [...]. Mystère ! Ce besoin d'aimer une femme n'a fait que se développer avec l'âge, il a traversé mes années de dévotion et de rigorisme, et à l'heure même où je t'écris, il me semble qu'une image féminine se joue sous mes yeux, que je vais la saisir ; ma plume court sur le papier, mes nerfs s'irritent, ma tête s'échauffe, je m'arrête [...] il m'en coûte tant de te faire un aveu si intime, je compte sur ta discrétion ${ }^{42}$.

Dans un climat de défiance, déjà évoqué, frappant le corps et le sexe au XIXe siècle, cette confession, douloureuse, est révélatrice de la réceptivité de l'enfant puis de l'adolescent : Materne, culpabilisé à l'extrême, présente sa conduite comme névrotique. L'aveu se conjugue ici avec la projection et la subtile mise en scène du « moi », qu'autorise le lien épistolaire.

Une fois établie, la relation intime entre deux condisciples prend rapidement des allures d'idylle, analogue, par bien des aspects, à celle pouvant exister entre un jeune homme et une femme. Le comportement amical emprunte, par exemple, de nombreux éléments au rituel de l'amour romantique. A en juger par les récits rétrospectifs, l'instant de la rencontre - sans atteindre au " coup de foudre » frappant le baron de Nucingen à la vue d'Esther dans Splendeurs et misères des courtisanes - est vécu assez intensément pour laisser un souvenir précis. Michelet, dans le Mémorial qu'il rédigea à l'intention de Poinsot, relate avec émotion « l'événement le plus important de ma vie [...] la manière dont j'ai connu Poinsot ; dès ce moment, je ne suis plus seul ${ }^{43}$. Le récit détaillé de la découverte de son futur ami, nouveau venu dans la pension Melot qu'il fréquentait alors (1811), montre à quel point la scène a été mémorisée.

L'attirance qu'éprouvent les futurs amis l'un pour l'autre semble immédiate, ou du moins très rapide. Michelet évoque une « reconnaissance » et Nisard « l'instinct. » De même, Cornudet croit fermement à la prédestination des êtres $^{44}$. Les liens se resserrent au gré de circonstances variées : Jules Barbey d'Aurevilly et Maurice de Guérin se trouvèrent « compagnons du même pupitre », dans la même classe d'étude du collège Stanislas, en 1827, ce qui ne pouvait que rapprocher ces deux sensibilités sceurs ${ }^{45}$.

Pour d'autres, la liaison fut directement encouragée par l'attitude persifleuse d'une partie de la communauté collégienne. Nisard et Trancart, fraîchement débarqués à Sainte-Barbe, en provenance, le premier, de Châtillon-sur-Seine, le second de Bar-le-Duc, déclenchèrent par leur attitude effarouchée, leur accent provincial et la coupe grossière de leurs habits, force quolibets et farces. La similitude de leur situation, l'infortune partagée de ce bizutage de potaches, trempa définitivement leur amitié : « Nous en souffrions ensemble, et nous nous en consolions plus vite " ${ }^{46}$. Dans ce même collège de Sainte-Barbe, quelques années plus tard (1827), c'est pour avoir trop ouvertement affiché leurs convictions chrétiennes que de Montalembert et Cornudet furent poursuivis par les railleries incessantes de leurs camarades impies. Cette épreuve intensifia une attirance réciproque : « tes pensées sur l'épanchement nécessaire entre des amis et sur la religion, qui comme tu le dis fort bien, a formé notre liaison, me semblent admirables » atteste, enthousiaste, Montalembert à Cornudet ${ }^{47}$.

Séparés généralement pendant la durée des classes et des études (environ une dizaine d'heures par journée), les amis n'avaient de cesse de se retrouver au plus vite. La cour de récréation s'imposait alors comme le lieu stratégique de possibles 
entrevues, « tête à tête, ou plutôt cœur à cœur " ${ }^{48}$. L'administration se méfiait de ces collégiens qui toujours allaient par deux, volontairement à l'écart des autres, scène imaginée par Balzac pour Louis Lambert et son compagnon : « Etrangers aux jouissances de nos camarades, nous restions seuls, mélancoliquement assis sous quelque arbre de la cour ${ }^{49}$. La teneur des conversations supposées dans ces circonstances, les excès d'imagination et les fâcheuses conséquences qui leur étaient associées engendraient les arlarmes les plus vives. Aussi ordonnait-on aux « pions » une surveillance de chaque instant : « Les conversations, les apartés, les retraites, la solitude, doivent être sévèrement bannis des récréations. Il faut jouer » ${ }^{\text {so. }}$.

De fait, soit intervention d'un surveillant, soit apparition inopportune d'un ou plusieurs camarades, il était malaisé de se parler longuement, au grand dam des confidents :

Notre conversation sentimentale, mon bien cher ami, a été fort mal à propos interrompue ce matin. Quand aurons-nous donc un jour, une heure à passer ensemble, cour à cour, comme tu le dis si bien, pour nous dire tout ce que nous sentons, tout ce que nous pensons, comme deux amis vrais et sachant s'entendre?

s'exaspère Léon Cornudet qui, interne comme son ami Montalembert mais dans la classe supérieure, n'a que peu d'autres occasions de le voir, sinon fugitivement $^{\mathrm{s1}}$.

Pour tromper la frustration de l'absence, le recours à la lettre s'impose naturellement et prolonge les délices de l'échange : « Et puisque nous ne pouvons pas trouver un pauvre petit moment pour rester seuls, puisque me voilà enfermé entre les murs étroits de ma cellule philosophique, je ne puis mieux faire, je ne puis trouver un plaisir plus doux que celui de m'épancher quelques instants dans ton cour » ${ }^{52}$. Assis côte à côte en étude, Barbey d'Aurevilly et Guérin ne s'en écrivaient pas moins de longues épîtres, bravant ainsi la surveillance du maître d'étude et négligeant leçons et devoirs. Le contenu strictement confidentiel de ce courrier - « quelques-uns de nos amis riraient peut-être s'ils voyaient nos lettres » présumait Cornudet ${ }^{\mathbf{5 2}}$-, les dangers encourus lors de la rédaction, les stratagèmes utilisés pour remettre la missive provoquaient des émotions semblables à celles ressenties par les amants clandestins qui se risquent à envoyer leur première déclaration d'amour : « Je tremblais en portant ma lettre chez toi, puis je me repentais de l'avoir envoyée. Je ne voyais point venir de réponse ! Est-ce une déclaration de guerre? Une rupture formelle ? Mon cœur palpite. Je lis les premières lignes et je suis heureux ! ${ }^{53}$.

La lettre , qui permet les confessions les plus achevées, favorise l'exaltation des sentiments et le lyrisme de leur formulation. La place essentielle qu'occupait la rhétorique dans l'enseignement de l'époque était sans doute aussi pour beaucoup dans cette propension qu'avaient les collégiens à laisser libre cours, dans leur correspondance, aux transports les moins mesurés ${ }^{54}$. Jules Simon, s'adressant à Gabriel Gallerand, rencontré au collège de Vannes, entrelace les « chéri » à un vouvoiement dont le raffinement atteint la préciosité ${ }^{55}$. Montalembert, usant de la langue anglaise comme d'un dernier rempart pour sa pudicité, appelle Cornudet « friend of my heart », celui-ci surenchérit alors par un « dearest friend of my heart » et se heurte aux reproches de son destinataire qui prétend à l'exclusivité dudit cœur. Leur correspondance, qui s'enflamme sous l'impulsion de 
Montalembert, prend, à bien des endroits, une tonalité amoureuse que ne renieraient pas les amants les plus épris : « une douce habitude m'a enchaîné à toi », « cette soif inextinguible que j'éprouve de te voir sans cesse, de te parler toujours », « il n'y a que nos deux cœurs qui se comprennent bien, qui s'aiment comme il faut, qui se confondent », « reviens-vite et aime-moi... comme tu m'aimes " ${ }^{56}$. Aimant à s'analyser, les jeunes gens aimaient aussi à se laisser deviner. Les vers que l'on rédige à l'intention de l'autre, les livres - préalablement annotés - que l'on prête ou que l'on offre, sont autant de façons de présenter, voilés, les tréfonds de son âme.

Dans les collèges, le comportement amical passe par l'exaltation du « moi ». Le goût des promenades solitaires, lors des rares congés et des vacances, plus rares encore, rend loisible le dialogue des cœurs : « ce sera alors surtout [dans nos délicieuses promenades] que mon cœur versera dans le tien ses peines et ses plaisirs et recevra de ces douces consolations une effusion de joie, une surabondance de joie inexprimable » écrit tendrement Ozanam à Materne ${ }^{57}$. Jules Simon, à la pensée de retrouver Gallerand, se réjouit de pouvoir reprendre leurs «promenades sentimentales " ${ }^{58}$. Michelet conte, dans son Mémorial, le bonheur des équipées à travers Paris, quand, flanqué de l'ami Poinsot, ils s'essayaient tous deux à suivre les filles ( $\ll$ nous étions des polissons vertueux » ${ }^{59}$ ). Barbey se remémore avec émotion les sorties du collège Stanislas, en compagnie de son inséparable Guérin :

Il est des endroits de Paris qui mont été consacrés par lui et des pierres ou du bitume desquels il sort une douce flamme pour mes yeux, quand je les revois. C'est là où nous avons échangé des sentiments et des pensées, fait de la vie, car il n'est pas d'autre mot pour exprimer cela ${ }^{60}$.

L'émotion, maîtresse incontestée de ces tête-à-tête, était courtisée de mille façons : les mots, les regards, les gestes mais aussi les silences, s'enchevêtraient pour mieux la capter et la retenir. La lecture des poètes, la contemplation partagée d'un paysage enchanteur engendraient des voluptés rapportées et ressassées indéfiniment entre les murs du collège.

Rivés l'un à l'autre par cette communion de l'âme, les périodes de séparation - « funeste image » proférait Montalembert ${ }^{61}$ - étaient vécues dans l'effroi et le chagrin. Au milieu de tous ses camarades, Montalembert se sent dans une « solitude entière ». Son esprit s'évade vers celui de son ami, pour se fondre en lui : « il est si doux, quand on se voit entouré d'une foule d'êtres indifférents, de se transporter pour ainsi dire dans l'âme de son ami, de s'isoler de son entourage pour s'identifier avec lui » ${ }^{62}$. Déjà, à la suite d'une cérémonie religieuse qui fouetta leurs élans spirituels, Cornudet avait soufflé : « Je devinais avant-hier ce qui se passait dans ton cœur ; mon âme était tout entière confondue avec la tienne; non n'étions plus qu'un, cher ami " ${ }^{63}$. De plus, même si la chaleur de ces amitiés ne garantit pas nécessairement leur pérennité, malgré les serments échangés par les intimes ${ }^{4}$, on constate le plus souvent que les liens étroits noués au collège entre condisciples évoluent, mais ne disparaissent pas au fil des années ${ }^{65}$.

L'analogie, partielle et imparfaite, entre les amis intimes et les amants, n'est pas le seul fait d'une analyse rétrospective. Elle s'imposait également à quiconque portait un regard sur ces tendres couples d'adolescents. François Chon, dans ses 
Impressions et souvenirs, décrit ces inséparables : « C'était chose amusante de les voir chuchotant ensemble, vrais amants par la tendresse et l'intimité ${ }^{66}$ \#. La hantise des déviations sexuelles (masturbation, homosexualité) poussait bientôt cette analogie à l'assimilation totale :

J'ai connu des pensions où ces amitiés, par l'ignorance et l'ineptie des maîtres, étaient devenues générales ; presque chaque élève avait sa Dulcinée dans un de ses camarades, soupirait pour lui, devanait son esclave, lui écrivait des billets les plus tendres : on devine les chutes nécessaires ${ }^{67}$.

Ce tableau, dressé par l'abbé Timon-David, pour exagéré qu'il soit, n'est pas dénué de toute vérité. Une homosexualité latente existe dans les collèges, particulièrement dans les internats. L'administration sévissait contre ce qu'elle considérait être une pratique ignominieuse. Ainsi, en 1836, un élève du collège royal de Poitiers, accusé d'avoir corrompu un "petit ", est exclu de l'établissement. La seule preuve tangible au dossier est cette lettre du jeune homme renvoyé, confisquée par un surveillant :

Cher Ange, Ton silence m'étonne au dernier point. Je ne sais à quoi l'attribuer. Je crains de t'avoir contrarié en quelque chose. Je t'en prie, mon tendre ami, écris-moi plus souvent. Occupé, comme du dois penser que je le suis, je n'ai pas beaucoup de temps à moi, cependant sois-sûr mon Victor, que tous mes moments de loisir seront pour toi, pour toi seul, pour te dire que je t'aime, que je t'adore! Oui mon ange, je t'adore ! J'espère que tu m'écriras au moins tous les huit jours. J'ai mis l'autre jour $R$. au défi devant plusieurs élèves de me faire voir aucune de tes lettres adressées à moi, il n’a jamais pu. Il a voulu aussi se mêler de faire courir certains bruits. Mais on ne l'a pas cru. Je crains bien que l'année ne se passera, sans que je lui flanque une bonne dégelée. Adieu mon ange. Quand tu me verras passer dans ta cour à midi et demi, demande à aller où je t'ai vu l'autre jour. Jai quelque chose à te donner. Ton tendre ami ${ }^{68}$.

La lecture de cette lettre manifeste toute l'influence qu'exercent sur la vie des jeunes gens les intimités ambiguês de collège. Ces liens participent grandement de l'élaboration d'une personnalité qui entre, à cet âge-là, dans une période décisive. Mais, surtout, ces amitiés exaltées, dans les confidences et les comportements qu'elles suscitent, permettent les rêves amoureux les plus doux. L'intimité masculine est bien, pour les collégiens, le premier chapitre de l'éducation sentimentale.

\section{NOTES}

1. Association des anciens élèves du collège Louis-Le-Grand, recueil de témoignages sur le college, sous forme de poésies et de chansons, Banquet annuel du 14 décembre 1850, imp. Crapelet, 1851. Les vers cités sont signés V. Betolaud, interne de 1811 à 1822 .

2. Sur l'enseignement, et les établissements scolaires, le livre de référence reste celui d'A. Prost, Histoire de l'enseignement en France 1800-1967, Armand Colin, 1968, auquel on ajoutera F. Mayeur, De la Révolution à l'école républicaine, t. 3 de l'Histoire générale de l'enseignement el de l'éducation en France, Nelle lib. de France, 1981. Voir également P. Gerbod, La Vie quotidienne dans les lycées et collèges au XIXe siècle, Hacheuc, 1968, et le livre ancien mais riche en ce qui concerme la vie dans les collèges, de G. Weill, Histoire de l'enseignement secondaire en France 1802-1920, Payot, 1921. 
3. In y a bien sûr des exceptions, comme celle d'E. Quinet : * Loin que mes compagnons de captivité fussent une consolation pour moi, j'eus presque autant de peine à m'accoutumer à eux

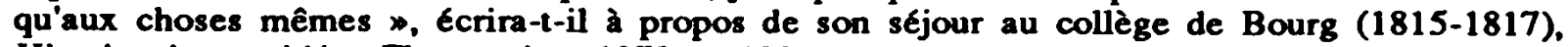
Histoire de mes idées, Flammarion, 1972, p. 123.

4. D. Nisard, Souvenirs et notes biographiques, Calmann-Lévy, 1888, p. 170-171. L'expérience de l'internat par Nisard remonte à la Restauration (collège Sainte-Barbe), mais celuici n'avait guère évolué jusqu'à la date de rédaction des Souvenirs. En évoquant les novateurs, Nisard pense d'abord à M. Bréal, professeur au Collège de France, dont l'ouvrage Quelques mots sur l'Instruction publique en Fance, Hachette, 1872, inspira la tentative de réforme des études classiques menée par Jules Simon.

5. Ibid., t. 1, p. 173.

6. A. Corbin, * La relation intime ou les plaisirs de l'échange $\nsim$, dans Histoire de la vie privée, Seuil, 1987, L 4 sous la direction de M. Perrot, De la Révolution à la grande Guerre, p. 514.

7. Abbe Masson, Le Miroir des collèges ou les vices effrayants de l'éducation universitaire sous le triple rapport du physique, de l'intelligence et du moral, Lecoffre, 1847 : * Il appartient à l'éducation de diriger [les penchants du cour] et de les incliner peu à peu vers le bien et la vertu [...] l'on doit dès le premier âge, s'occuper avec un soin extrême de développer les qualités du coeur d'où dérivent les sentiments honnêtes et nobles ; seul il fait le fils respectueux, le bon époux, le bon père et l'homme de mérite dans les lettres, les sciences et les arts $\nsim$ p. 148. L'absence d'éducation morale dans les établissements publics était l'un des arguments favoris de l'Eglise dans son réquisitoire contre l'Instruction publique. Cf. Abbé Masson, p. 149-150.

8. Pour le Dr Pavet de Courteille, Hygiène des collèges et des maisons d'éducation, Gabon, 1827, il s'agit toujours de * façonner ces jeunes coeurs * (p. 117), mais la finalité diverge : * Si l'enfant que l'on destine à recevoir au collège les bienfaits de l'éducation publique a été préparé de bonne heure par une main industrieuse et chère [...], s'il a déjà appris à vivre en société, il sera tranquille, heureux, content dans ce nouveau séjour $\star$. p. 118-119.

9. M. Foucault, La Volonté de savoir, Gallimard, 1976, p. 137-138.

10. Dr Deslandes, De l'onanisme et des autres abus vénériens considérés dans leurs rapports avec la santé, Lelarge, 1835, p. 506. Cf. également J. E. Dufieux, Nature et virginité, Lanier, 1854 , p. 146-147.

11. J. J. Rousseau, Emile ou de l'éducation, Garnier - Flammarion, 1966, p. 115. C. Petitfrère a rappelé cette peur que le pouvoir domestique suscite chez l'enfant, dans $L^{\prime} C$ Eil du maître. Maîtres et serviteurs, de l'époque classique au romantisme, Complexe, 1986, p. 154-157.

12. Dr Deslandes, o. c., p. 508.

13. J. Barbey d'Aurevilly, « Le Buste jaune $\star$ dans * Poussières $\star$ (poésies), CEuvres romanesques complètes, \& La Pléiade $\star, 1966, t$, 2, p. 1187-1189. Il existe une reproduction photographique de ce buste, représentant Mme de Chavincourt, grand-tante de Barbey, dans J. P. Seguin, Inconographie de Barbey d'Aurevilly, Genève, Cailler, 1961.

14. R. H. Guerrand, Histoire de la vie, privée, t. 4, « Espaces privés », p. 334.

15. J. Michelet, Mémorial dans Ecrits de jeunesse, Édition P. Viallaneix, Gallimard, 1959, p. $188-189$.

16. Histoire de la vie privée, t. 4, « Drames et conflits familiaux $*$ p. 272. Cf. Egalement C. Bonnello, Le Disours médical sur l'homosexualité en France au XIXe siècle. Thèse de 3ème cycle d'histoire sous la direction de M. Perrot, Paris 7, 1987 : * Il ne s'agit plus tant de protéger à court terme, même si ceute préccupation demeure, la jeunesse des méfaits du sens génital [discours sur l'onanisme et moyens de le combattre] mais, à travers elle [la jeunesse], protéger le renouvellement de la société et de la race $\%$, p. 388.

17. Histoire de la vie privée, L. 4, * Le secret de l'individu ». p. 454.

18. Abbe Masson o. c., p. 366. Cf. aussi Dr Lallemand, Des pertes séminales involontaires, Béchet, 1836, 3 vol., p. 318 du t. 1 . AP 1).

19. Lettre de Saint-Marsal a Théodore Ducos, 20 janvier 1820, Archives Nationales (46

20. * Révolution * : le mot le plus employé pour désigner la puberté dans les thèses de médecine. Cf N. Azri, La Puberté dans le discours médical et la société (fin XVIIIe début XIXe siècles), mémoire de man̂rise d'Histoire sous la direction de M. Perrot, Paris 7, 1985, p. 35.

21. Norben Elias, Uber den Prozess der Zivilisation, traduit en français sous le titre de $L a$ Civilisation des moeurs, Calmann-Lévy, col. * Pluriel », 1982, p. 304. 
22. Cf l'autobiographie de J. Favre, Henry Belval, Typographie Chamerot, 1880 ; J. Favre s'y raconte, sous couven du protagoniste Henry Belval : « Quant à d'autres plaisirs qui, avec sa nature impétueuse, pouvaient lui être mortels, son éducation en avait à l'avance émoussé l'autrait, moins par son austérité que par la délicatesse des sentiments qu'elle lui avait inculqués. La direction de Mme Belval, sur ce point si grave et si difficile, avait été aussi ferme qu'éclairée : partant de l'idée chrétienne, et peut-être aussi ferme qu'éclairée ; partant de l'idée chrétienne, et peut-être l'exagérant, elle avait de bonne heure accoutumé son fils à observer avec une grande sévérité les lois de la décence. Elle lui répétait souvent que nous devons respecter notre corps dont nous sommes responsables devant Dieu; elle lui faisait remarquer, par des exemples discrètement choisis, tout ce que la pudeur donne aux femmes de grâce et d'autorité, tout ce que la violation de ses lois enlève trop souvent de valeur véritable aux hommes. Elle combattait éloquement la distinction injuste et corruptrice que les mours ont créé à cet égard entre les deux sexes, et ne cessait de répéter que la chasteté n'était pas seulement une ventu, qu'elle était la plus exquise des beautés, le talisman le meilleur pour être vraiment aime $»$, p. 58.

23. J. et E. de Goncourt, Journal. Mémoires de la vie littéraire, édition R. Ricatte, Collection * Bouquins, 1989, t. III, p. 301 (21-22 juillet 1889). C'est Edmond qui trouva à Jules sa première maîtresse, la sienne même, après que Jules lui eut confié ses déboires avec une protituée.

24. L'enseignement public, l'instar du prive, inscrit ostensiblement son action dans le prolongement de celle de la famille : * Il est un principe fondamental que les instituteurs ne doivent jamais perdre de vue, c'est que substitués à la sollicitude patemelle, ils sont véritablement investis, à l'égard de leurs élèves, d'une mission non moins sacrée, non moins étendue que celle du père de famille * (circulaire aux proviseurs des collèges royaux, 5 octobre 1822). En 1836, c'est le ministre Pelet de la Lozère qui en appelle au sentiment de patemité des professeurs, qui doivent avoir à coeur d'inculquer aux enfants * des affections de famille $\star_{,}$* des vertus domestiques $*$ avant même les \& vertus sociales * (circulaire aux recteurs, 7 juillet 1836).

25. Dr Pavet de Courteille, o. c., p. 155.

26. G. Flaubert, Correspondance, ed. J. Bruneau, « La Pléiade «, 1973, 2 vol. parus, leure du 12 août 1857 , p. 756-757 du L. 2.

27. F. Chon, Impressions et souvenirs 1812-1872, Lille, imp. Danel, 1882, p. 137.

28. L. G. Taillefer, De quelques améliorations d introduire dans l'Instruction publique, Renouard, 1824, p. 74.

29. Leture de Femand de Sassenay à sa mère, 2 novembre 1846, Archives Nationales (337 AP 17).

30. Ed. cit., * Figures et rôles $\nsim$, p. 163.

31. Charles de Montalembert (1810-1870) / Léon Comudet (1808-1876) : condisciples au collège Sainte-Barbe. Voir leur correspondance : Lettres d un ami de collège 1827-1830, Lecoffre, 1884. (La correspondance a été hélas expurgée du * trop intime * à la publication.) Lettre du 5 juin 1827, p. 4.

32. Frédéric Ozanam (1813-1853) / Auguste Materne (1812-1893) : condisciples au collège royal de Lyon en 1830. Voir leur correspondance dans F. Ozanam, Lettres, Bloud, 1971, 3 vol. ; t. 1, p. 15, leture du 5 juin 1830.

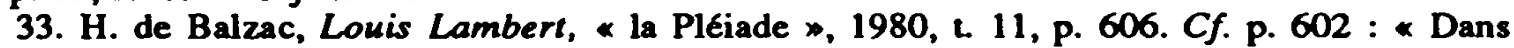
notre langage collégial, le mot être faisants (ailleurs, c'est copins) constituait un idiotisme difficile à traduire. Il exprimait un partage fratemel des biens et des maux de notre vie enfantine, une promiscuité d'intérêts fertile en brouilles et en raccommodements, un pacte d'alliance offensive et défensive.

34. J. Michelet, Mémorial, éd. cit, p. 192 et p. 194.

35. Souvenirs el noles biographiques, éd. cit., p. 181.

36. Lettres d̀ un ami de collège, éd. cit., p. 8, leure datée de 1827.

37. F. Ozanam, Lettres, éd. cit., p. 23, lettre datée de juin 1830.

38. Ibid., lettre du 5 juin 1830, p. 14 et p. 22 pour la seconde.

39. Voir par exemple J.P. Sartre, L'Idiot de la famille, Gallimard, 1988, 3 vol., t. 1. p. 1009 et suiv. : la correspondance de Flaubert, en particulier celle adressée à Emest Chevalier et Alfred Le Poituevin, et l'éude de R. Kempf sur l'amitié de Flaubert et Le Poittevin : * Le double pupitre », Les Cahiers du chemin, oct. 1969, p. 122-149.

40. La Dynamique de l'Occident, Calmann-Lévy, 1975, p. 270. suiv.

41. Cf. les superbes pages de J.P. Sartre à ce propos, L'Idiot de la famille, t.2, p. 1382 et 
42. Lettre d'A. Materne H. Fortoul du 13 juillet 1831, Archives Nationales (246 AP 4).

43. J. Michelet, Mémorial, éd. cit., p. 191.

44. J. Michelet, Mémorial p. 192 ; D. Nisrd, Souvenirs et notes biographiques, p. 180 ; Lettres dun ami de collège, éd. cit., Cornudet à Montalembert.

45. Jules Barbey d'Aurevilly (1808-1889) / Maurice de Guérin (1810-1839) : condisciples au collège Stanislas en 1827. Voir leurs correspondances respectives : J. Barbey d'Aurevilly. Correspondance générale, Les Belles Lettres, 1980, 9 vol. parus; M. de Guérin, Correspondance dans Euvres complètes t. 2, Les Belles Lettres, 1947. Ici Barbey, Corr., t. 4, p. 252, lettre à Trébutien, 15 août 1855.

46. Souvenirs et notes biographiques, o. c., p. 181.

47. Lettres d̀ un ami de collège, ed. cit., leture de juin 1827, p. 11.

48. Ibid., p. 5 , leture du 5 juin 1827.

49. Lowis Lambert, ed. cit., p. 613.

50. Dr Pavet de Courteille, o.c., p. 96. A en croire Victor de Laprade, regreuant l'inertie des collèges en récréation, ces conseils n'étaient guère suivis : L'Education homicide. Plaidoyer pour l'enfance, Didier et Cie, 1868, p. 44.

51. Lettres à un ami de collège, éd. cit., leure de Comudet à Montalembert de 1827, p. 6-7.

52. Ibid., p. 8.

53. Ozanam, Lettres, éd. cit., p. 16, leure du 8 juin, 1830.

54. La rhétorique ne se réduit pas toutefois à cet apprentissage juvénile du lyrisme, puisque l'on sait que les codifications strictes qu'elle met en jeu apprennent aussi au jeune homme le contrôle sourcilleux de ses mots, de ses attitudes et de ses actes.

55. Voir la correspondance de Jules Simon (1814-1896) aux Archives Nationales (87 AP 8).

56. Lettres du un ami de collège, éd. cit., lettres à Cornudet du 5 juin 1827 (p. 5), du 8 août 1827 (p. 26), du 31 août 1827 (p. 37) et du 12 oct. 1827 (p. 78).

57. Lettres, éd. cit., juin 1830, p. 23.

58. Leure de J. Simon à Gallerand du 6 juillet 1833, Archives Nationales (87 AP 8).

59. Ed. cit., p. 192.

60. Correspondance générale, t. 4, p. 252, à Trébutien, 15 août 1855.

61. Lettres d̀ un ami de collège, éd. cit., p. 33.

62. Ibid., lettre à Comudet du 12 oct. 1827, p. 78.

63. Ibid., leture Montalembert, août 1827, p. 21.

64. Lettres d un ami de collège, p. 76, De Comudet à Montalembert, 7 oct. 1827 : \& Nous sommes l'un pour l'autre à la vie, à la mort *. Ozanam à Mateme (Lettres, éd. cit, 8 juin 1830, p. 16) : * Nous nous aimerons toute notre vie et notre belle religion nous dit que nous nous aimerons encore au delà $\star$.

65. Si les liens se distendent entre Ozanam de Materne, au sortir du collège, Barbey d'Aurevilly et Guérin restèrent intimes jusqu'à la mort du second en 1839. De la même façon, la mort mit fin à l'amitié de Poinsot (décédé en 1821) et de Michelet. Trancart et Nisard, Montalembert et Cornudet entretinrent une amitié très vive tout au long de leur vie. Voir Souvenirs et notes biographiques, o.c., p. 181 et suiv. ; Montalember / Cornudet, Correspondance 1831-1839, Champion, 1905.

66. F. Chon, o. c., p. 213.

67. Abbe Timon-David, Traité de la confession des enfants et des jeunes gens, Sarlit, 1865, 2 vol. ; L. 2, p. 231.

68. Lettre cité par J. Contou, Les Punitions dans les lycées et collèges de l'Instruction publique en France 1814-1854, Thèse de 3ème cycle, sous la direction d'Antoine Léon, Paris V. juin 1980, 2 vol. ; t. 1, p. 118. 